\title{
Lattice-shifted nematic quantum critical point in $\mathrm{FeSe}_{1-x} \mathrm{~S}_{x}$
}

\author{
S. Chibani (iD) ${ }^{1}$, D. Farina (D) ${ }^{1}$, P. Massat ${ }^{1}$, M. Cazayous ${ }^{1}$, A. Sacuto (D) ${ }^{1}$, T. Urata ${ }^{2,9}$, Y. Tanabe ${ }^{2,3}$, K. Tanigaki ${ }^{2,4}$, A. E. Böhmer ${ }^{5,6,10}$ \\ P. C. Canfield ${ }^{6,7}$, M. Merz iD $^{5}$, S. Karlsson ${ }^{8}$, P. Strobel ${ }^{8}$, P. Toulemonde ${ }^{8}$, I. Paul ${ }^{1}$ and Y. Gallais (iD ${ }^{1 凶}$
}

We report the evolution of nematic fluctuations in $\mathrm{FeSe}_{1-x} \mathrm{~S}_{x}$ single crystals as a function of Sulfur content $x$ across the nematic quantum critical point (QCP) $x_{c} \sim 0.17$ via Raman scattering. The Raman spectra in the $B_{1 \mathrm{~g}}$ nematic channel consist of two components, but only the low energy one displays clear fingerprints of critical behavior and is attributed to itinerant carriers. Curie-Weiss analysis of the associated nematic susceptibility indicates a substantial effect of nemato-elastic coupling, which shifts the location of the nematic QCP. We argue that this lattice-induced shift likely explains the absence of any enhancement of the superconducting transition temperature at the QCP. The presence of two components in the nematic fluctuations spectrum is attributed to the dual aspect of electronic degrees of freedom in Hund's metals, with both itinerant carriers and local moments contributing to the nematic susceptibility.

npj Quantum Materials (2021)6:37; https://doi.org/10.1038/s41535-021-00336-3

\section{INTRODUCTION}

The link between quantum criticality and the emergence of unconventional superconducting (SC) states is ubiquitous among several families of materials including heavy fermion, cuprates, and iron-based (Fe SC) superconductors ${ }^{1-3}$. The presence of divergent critical fluctuations associated to a nearby order are thought to provide a pairing glue and significantly enhance the SC transition temperature ${ }^{4,5}$. Dating from the seminal work of Berk and Schrieffer ${ }^{6}$, most studies have been devoted to fluctuations near a magnetic instability, and in particular antiferromagnetic (AF) ones which give rise to non s-wave pairing states such as d-wave in the cuprates, and possibly in heavy fermion and organic $\mathrm{SC}^{7}$.

In many Fe SC, SC emerges on the border of an AF state that is, however, also accompanied, or even preceded, by an electron nematic phase whereby the electron fluid spontaneously breaks the fourfold rotational symmetry of the underlying tetragonal lattice $^{8,9}$. The initial focus was on the proximity of the AF phase which is expected to lead a sign changing $\mathrm{s}^{ \pm}$SC pairing state ${ }^{10,11}$, but recently the role of nematic fluctuations and criticality on the SC state has come under scrutiny ${ }^{12-20}$. Several theoretical works have argued that the SC pairing is generically enhanced near a nematic quantum critical point $(\mathrm{Q} C P)^{5,21-24}$, even if it is not the leading pairing glue. On the experimental side, disentangling the role of $\mathrm{AF}$ and nematic QCP has proved challenging because the associated orders are essentially concomitant in most Fe SC. An exception is FeSe, where at ambient pressure SC emerges at about $8 \mathrm{~K}$ out of a nonmagnetic nematic state that sets in at much higher temperature $\sim 90 \mathrm{~K}^{25,26}$. The origin of the nematic state in FeSe is currently under debate, with both orbital and spin degrees of freedom possibly playing a role ${ }^{27-31}$. Weakening the nematic order in order to reach a putative QCP can in principle be achieved by either physical or chemical pressure. In the case of hydrostatic pressure, a magnetic order sets in before the end point of the nematic phase precluding a nematic $\mathrm{QCP}^{32-35}$. By substituting selenium (Se) with isovalent sulfur $(\mathrm{S})$, however, the nematic order can be continuously suppressed without any magnetic order $^{15,36,37}$ (see Fig. 1a), providing a model system to study the impact of a nematic QCP on SC and normal state properties.

Several studies indicate a significant impact of the nematic order on the SC pairing state ${ }^{38-41}$. However, intriguingly the evolution of $T_{c}$ in $\mathrm{FeSe}_{1-x} \mathrm{~S}_{x}$ indicates a marginal role for nematic quantum critical fluctuations in boosting SC. In fact coming from the tetragonal, non-nematic side of the $\mathrm{FeSe}_{1-x} \mathrm{~S}_{x}$ phase diagram, $T_{c}$ is essentially flat upon approaching the nematic QCP at $x_{c} \sim$ 0.17 showing only a mild maximum well-inside the nematic ordered phase ${ }^{42,43}$ (Fig. 1a). This apparent contradiction with theoretical expectations was recently argued to arise from the coupling between electronic nematic degrees of freedom and the lattice, which cuts off nematic quantum criticality at low temperature. This effect may suppress the expected enhancement of $T_{c}$ found in electronic-only models ${ }^{24}$. The lattice may also play a significant role in restoring Fermi liquid behavior of the normal state near the QCP ${ }^{44-46}$. In general, the effect of the lattice appears to have been overlooked in several previous studies of the nematic QCP FeSe $\mathrm{Fe}_{1-x} \mathrm{~S}_{x}{ }^{15,47}$, calling for an experimental clarification of its potential role in the properties of $\mathrm{FeSe}_{1-x} \mathrm{~S}_{x}$ near the nematic end point.

Here we use the ability of Raman scattering to probe symmetry resolved electronic fluctuations in the long wavelength limit $(q=$ $0)^{48-55}$ to investigate the evolution of nematic fluctuations in $\mathrm{FeSe}_{1-x} \mathrm{~S}_{x}$ as a function of doping and temperature. Our study spans a significant portion of the phase diagram of $\mathrm{FeSe}_{1-x} \mathrm{~S}_{x}$ from $x=0$ to $x=0.27$. This allows us to assess the evolution of critical nematic fluctuations across the nematic QCP, located at $x_{c}$ $\sim 0.17^{39,42}$. The dynamical nematic fluctuations spectrum consists of two main components, but only one of them shows a clear critical behavior upon approaching the nematic phase transition. A Curie-Weiss analysis of the critical component reveals a significant shift between the bare electron-only nematic QCP that is captured by Raman scattering, and the thermodynamic one. We

\footnotetext{
${ }^{1}$ Université de Paris, Matériaux et Phénomènes Quantiques, CNRS, Paris, France. ${ }^{2}$ Department of Physics, Graduate School of Science, Tohoku University, Sendai, Japan. ${ }^{3}$ Department of Applied Science, Okayama University of Science, Okayama, Japan. ${ }^{4}$ Advanced Institute for Materials Research, Tohoku University, Sendai, Japan. ${ }^{5}$ Karlsruhe Institute of Technology, Institute for Quantum Materials and Technologies, Karlsruhe, Germany. ${ }^{6}$ Ames Laboratory US DOE, lowa State University, Ames, IA, USA. ${ }^{7}$ Department of Physics and Astronomy, lowa State University, Ames, IA, USA. ${ }^{8}$ Université Grenoble Alpes, CNRS, Grenoble INP, Institut Néel, Grenoble, France. ${ }^{9}$ Present address: Department of

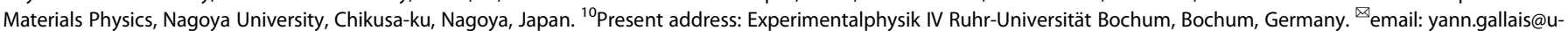
paris.fr
} 
(a)

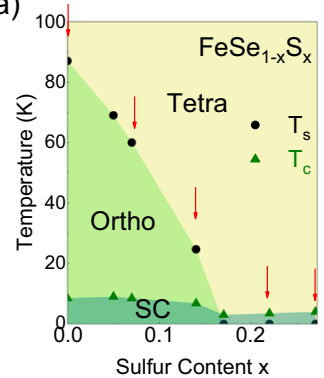

(b)

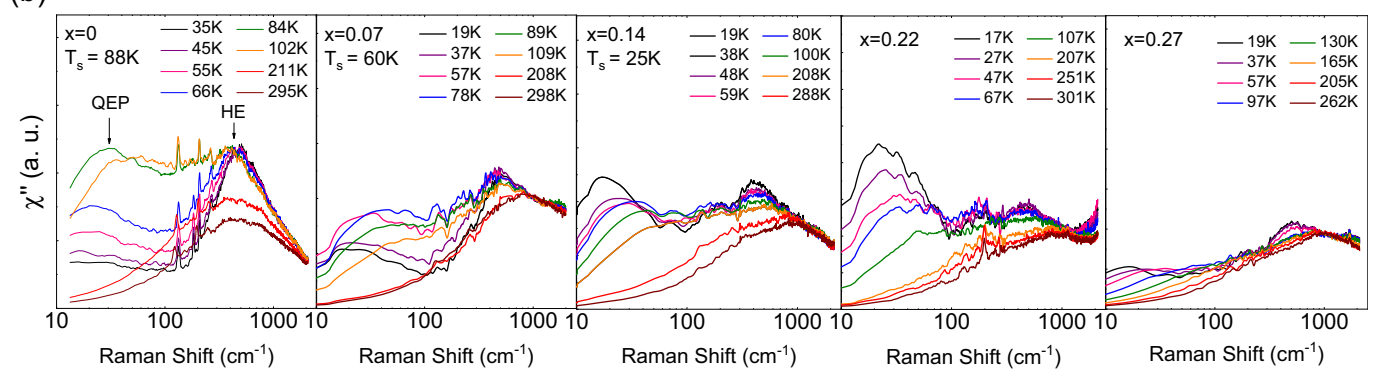

Fig. 1 Raman spectra in the $\boldsymbol{B}_{1 \mathbf{g}}$ nematic channel of $\mathrm{FeSe}_{\mathbf{1}_{-\boldsymbol{x}}} \mathbf{S}_{\mathbf{x}}$. a Phase diagram of $\mathrm{FeSe}_{1-x} \mathrm{~S}_{x}$. The arrows indicate the compositions whose Raman spectra are shown in (b). b Raman response as a function of temperature in $B_{1 \mathrm{~g}}$ symmetry channel for five different sulfur compositions. The energy is shown on a logarithmic scale. The quasi-elastic peak (QEP) and high-energy peak (HE) are indicated by arrows in the $x=0$ spectra.

attribute this shift to electron-lattice coupling, whose energy scale is found to be a sizable fraction of the typical Fermi energy of $\mathrm{S}-\mathrm{FeSe}$, and may explain the absence of $T_{c}$ enhancement at the nematic QCP. Our study highlights the important role of electron-lattice coupling effects in both SC and normal state properties of FeSe, and more generally of Fe SC.

\section{RESULTS}

\section{Sulfur doping dependence of symmetry-resolved Raman spectrum}

In our study, seven different sulfur (S) compositions $x$ were studied. The $\mathrm{S}$ content of the studied single crystals, which usually differs significantly from the nominal one, was determined by element-specific energy dispersive X-ray spectroscopy (EDS) and single-crystal X-Ray Diffraction (XRD) measurements (see "Methods"). The structural/nematic transition $T_{s}$ was determined by transport measurements on crystals from the same batch, and also in situ via the observation of the onset of elastic light scattering by nematic domain formation below $T_{s}$ (see Supplementary Fig. 1). $T_{c}$ was determined by the onset of diamagnetic signal in SQUID magnetometry. The phase diagram in Fig. 1 summarizes the properties $\left(x, T_{s}, T_{c}\right)$ of the crystals studied. Details on the Raman scattering measurements can be found in the "Methods" section.

In Fig. 1b, we show the temperature dependence of the electronic Raman scattering spectrum for five different dopings $(x=0, x=0.07(2), x=0.14(2), x=0.22(2)$ and $x=0.27(2))$ over a relatively wide energy range $\left(0-2000 \mathrm{~cm}^{-1}\right.$, corresponding to $0-250 \mathrm{meV}$ ). Data for $x=0$ (FeSe) were already reported in $^{52}$. Incoming and outgoing photon polarizations were oriented so as to probe the $B_{1 \mathrm{~g}}$ symmetry channel (using the $1 \mathrm{Fe}$ unit cell notation), which transforms as $x^{2}-y^{2}$ and thus corresponds to the nematic order parameter symmetry (see "Methods"). For all compositions, two main features, marked by arrows in the $x=$ 0 spectra, can be distinguished: a relatively broad peak at high energy (hereafter labeled HE peak) located at around $400 \mathrm{~cm}^{-1}$ $(50 \mathrm{meV})$, and a narrower feature, a quasi-elastic peak (QEP), located at much lower energy, below $100 \mathrm{~cm}^{-1}(\sim 12 \mathrm{meV})$ at low temperatures. The low $x$ content data are consistent with a previous Raman study which reported data in a more limited spectral and doping range ${ }^{56}$ (see Supplementary Discussion for a comparison between the present work and ref. ${ }^{56}$ ). The HE peak intensity decreases with doping (see also Fig. 2) and is only mildly affected by $T_{s}$. The QEP by contrast is strongly affected by $T_{s}$ and displays a significant doping and temperature dependence: its intensity is maximum close to $T_{s}$ for lower $x$ compositions, and continuously increases down to the lowest measured temperature $(17 \mathrm{~K})$ for $x=0.22$. The enhancement and collapse of the QEP across the nematic/structural transition temperature is consistent with previous studies in various $\mathrm{Fe} \mathrm{SC}^{49,51,52,55}$ and is ascribed to critical nematic fluctuations near $T_{\mathrm{s}}$. The QEP intensity is significantly reduced for $x=0.27$ (see Figs. $1 \mathrm{~b}$ and 2) indicating a reduction of nematic fluctuations for high $x$ content. Figure 2 shows the symmetry dependence of the QEP and HE peak: consistent with the nematic fluctuations interpretation, the QEP is absent in both $B_{2 g}$ and $A_{1 \mathrm{~g}}$ channels throughout the compositional range studied. Interestingly, the HE peak displays a robust symmetry dependence: it is observed only in the $B_{1 \mathrm{~g}}$ channel for all $x$.

\section{Extracting critical nematic fluctuations}

In order to analyze in more details the two main components of the nematic fluctuation spectrum, we performed a two component fit of the Raman spectra, where the QEP and HE peaks are fitted by an overdamped Lorentzian and a damped oscillator, respectively. $x^{\prime \prime}(\omega)=A \frac{\omega \Gamma_{\mathrm{QEP}}}{\omega^{2}+\Gamma_{\mathrm{QEP}}^{2}}+B \frac{\omega \omega_{\mathrm{HE}}^{2} \Gamma_{\mathrm{HE}}}{\left(\omega \Gamma_{\mathrm{HE}}\right)^{2}+\left(\omega^{2}-\omega_{\mathrm{HE}}^{2}\right)^{2}}$. A small linear background was added to model the high energy part of the spectra. We discuss the possible origin the HE peak below. The overdamped functional form taken for the QEP can be linked to a Drude-like Raman response of itinerant carriers that is renormalized near a nematic instability. In this framework, $\Gamma_{\text {QEP }}$ is an effective quasiparticle scattering rate where the impurity scattering rate $\Gamma_{0}$ in the $B_{1 \mathrm{~g}}$ channel is renormalized by the nematic correlation length $\xi_{n}$ : $\Gamma_{\text {QEP }}=\Gamma_{0}\left(\frac{a}{\xi_{n}}\right)^{2}$ where $a$ is the lattice parameter of the Fe plane $50,57,58$. We note that an alternative description in terms of coupling of itinerant carriers to finite momentum stripe-like spin-nematic fluctuations via Aslamasov-Larkin processes yields essentially the same functional lineshape for the QEP, making it difficult to conclude on the exact mechanism of the QEP ${ }^{59-61}$. The two approaches differ in the way the Raman probe couples to nematic degrees of freedom, but they both associate the QEP to the response to itinerant carriers near a nematic instability. On the other hand, the QEP lineshape cannot be accounted by theories of the Raman response based on local spin physics alone ${ }^{62}$

As shown in Fig. 3a (see Supplementary Fig. 3 for more doping and temperatures), all the spectra below $\sim 150 \mathrm{~K}$ could be reproduced with this decomposition. At higher temperatures, the QEP and HE essentially merge, making it difficult to unambiguously separate them via the fitting procedure. The HE peak energy was found to be weakly dependent of doping and temperature, but its width $\Gamma_{\mathrm{HE}}$ displays a marked decrease below $T_{s}$ (see Supplementary Fig. 5). From the decomposition, we can extract the behavior of the static nematic susceptibility $X_{0}$ associated to each component (Fig. 3b). Indeed using Kramers-Kronig relations, we have: $x_{0}^{\mathrm{QEP}}=\int_{0}^{\infty} \frac{x^{\prime \prime}}{\omega} d \omega=A$ and equivalently $X_{0}^{\mathrm{HE}}=B$. For all dopings the QEP component is the dominant contribution to the nematic susceptibility. Besides, as shown in Fig. 3b, the QEP component is significantly more critical 

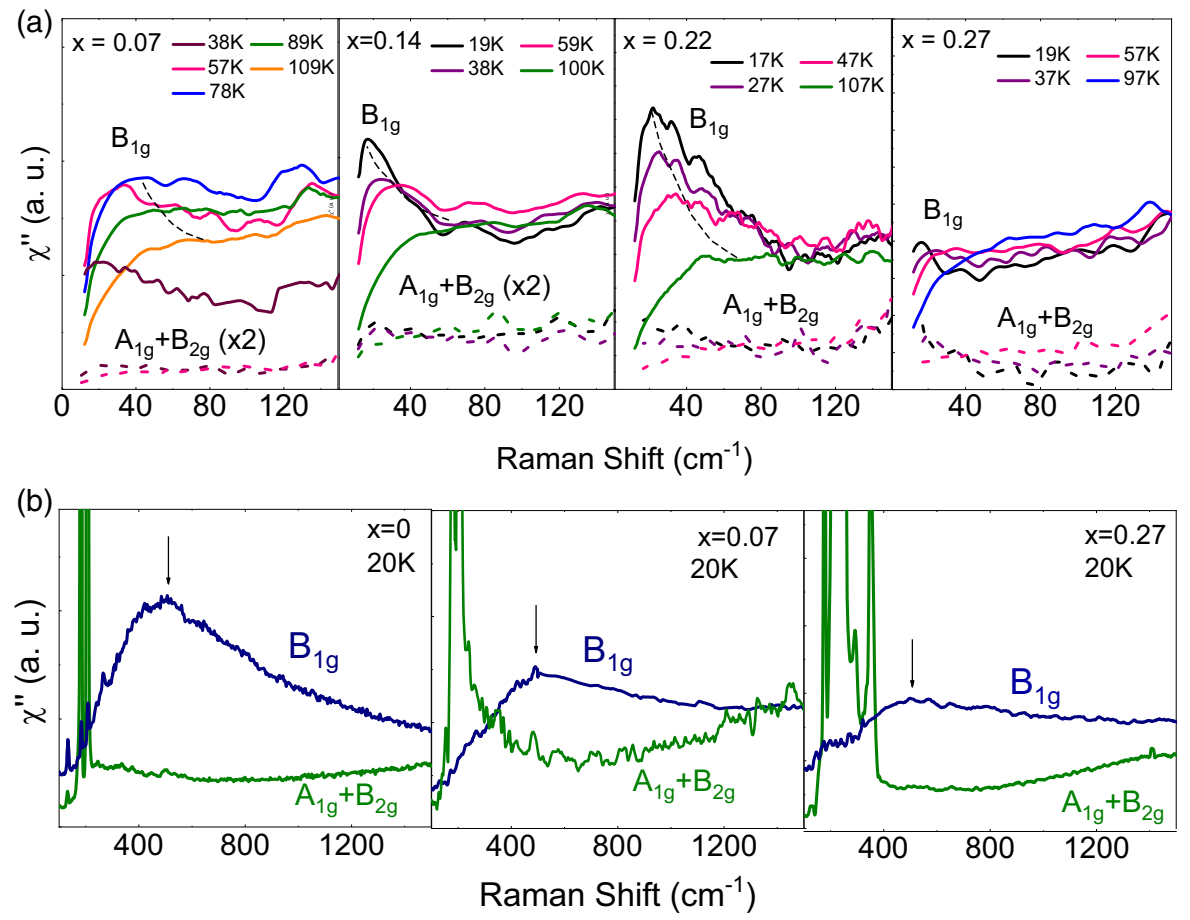

Fig. 2 Symmetry dependence of the Raman spectra. a Low energy part (on a linear scale) of the Raman spectra for two different symmetry channels $B_{1 \mathrm{~g}}$ (full lines) and $A_{1 \mathrm{~g}}+B_{2 \mathrm{~g}}$ (dotted lines) and for $x=0.07, x=0.14, x=0.22$, and $x=0.27$. $\mathbf{b}$ symmetry channel dependence of the spectra over a wide energy range on a linear scale for $x=0, x=0.07$, and $x=0.27$.
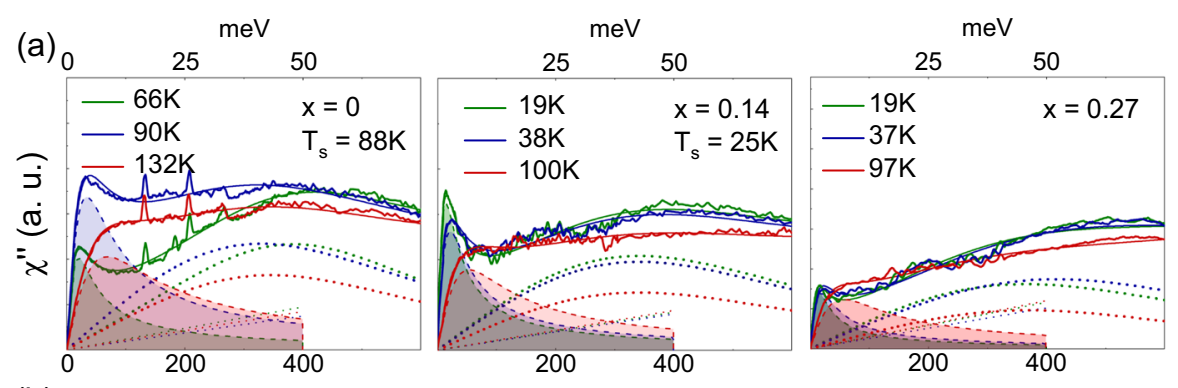

(b)

Raman Shift $\left(\mathrm{cm}^{-1}\right)$

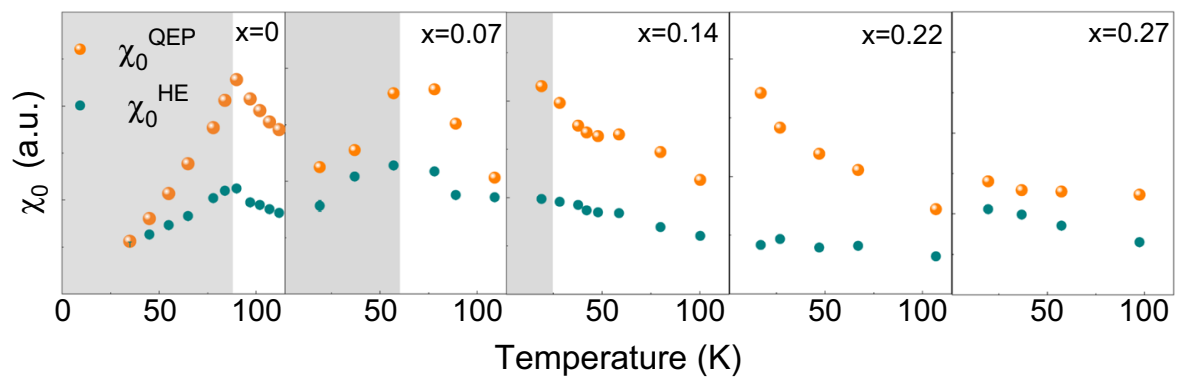

Fig. 3 Two component analysis of the nematic fluctuation spectrum. a Decomposition of the $B_{1 \mathrm{~g}}$ Raman response illustrated at three different compositions: $x=0, x=0.14$, and $x=0.27$ (see equation in the main text). The QEP peak component is highlighted via shading. b Temperature dependence of the two main contributions (QEP and HE) to the static nematic susceptibility $X_{0}$. Standard errors of the fits are shown but are usually smaller than the symbols.

than the HE component (see also Supplementary Fig. 7). An exception is $x=0.27$ where both contributions display a similar mild temperature dependence. This points to two distinct sources of nematic fluctuations in FeSe, with one displaying a more critical behavior near the nematic QCP.

We note that a different decomposition of the Raman response of FeSe was proposed $\mathrm{in}^{56}$, where a gap-like suppression was added in order to reproduce the loss of intensity of the spectra at low energy in $B_{1 \mathrm{~g}}$ symmetry below $T_{s}$. The gap opening was interpreted as the consequence of a modulated orbital order. In our case, we found that the spectra across $T_{s}$ could be well reproduced without invoking a gap. Rather the apparent spectral gap below $T_{s}$ results in our case from a combination of the collapse of the QEP contribution in the nematic phase and a sizable reduction of the width of the $\mathrm{HE}$ peak. The HE peak being present at all doping and 
(a)

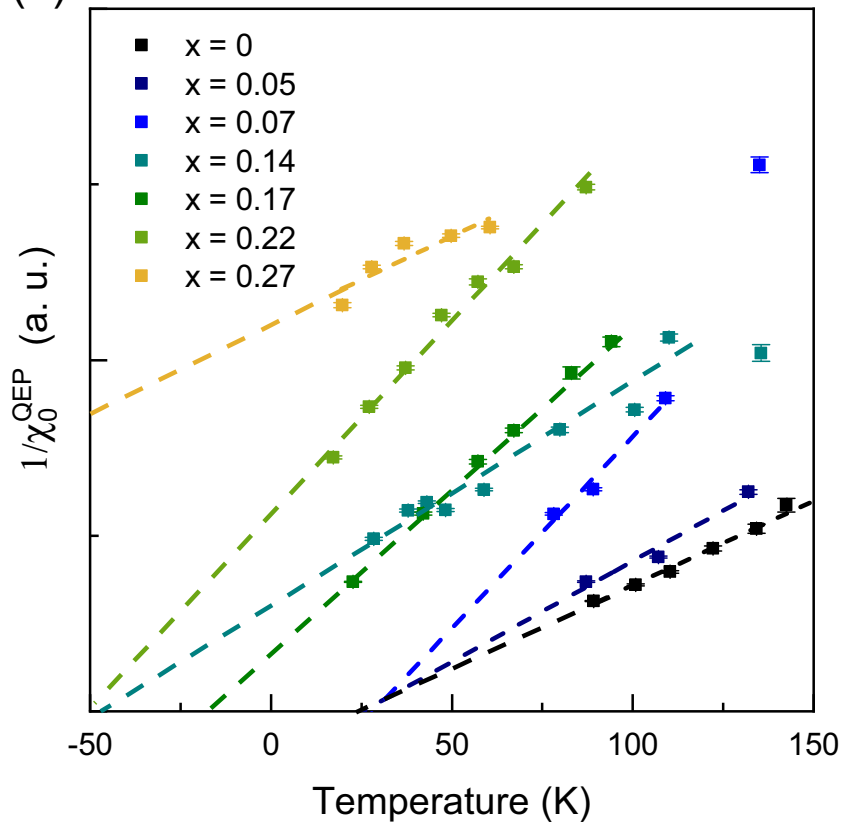

(b)

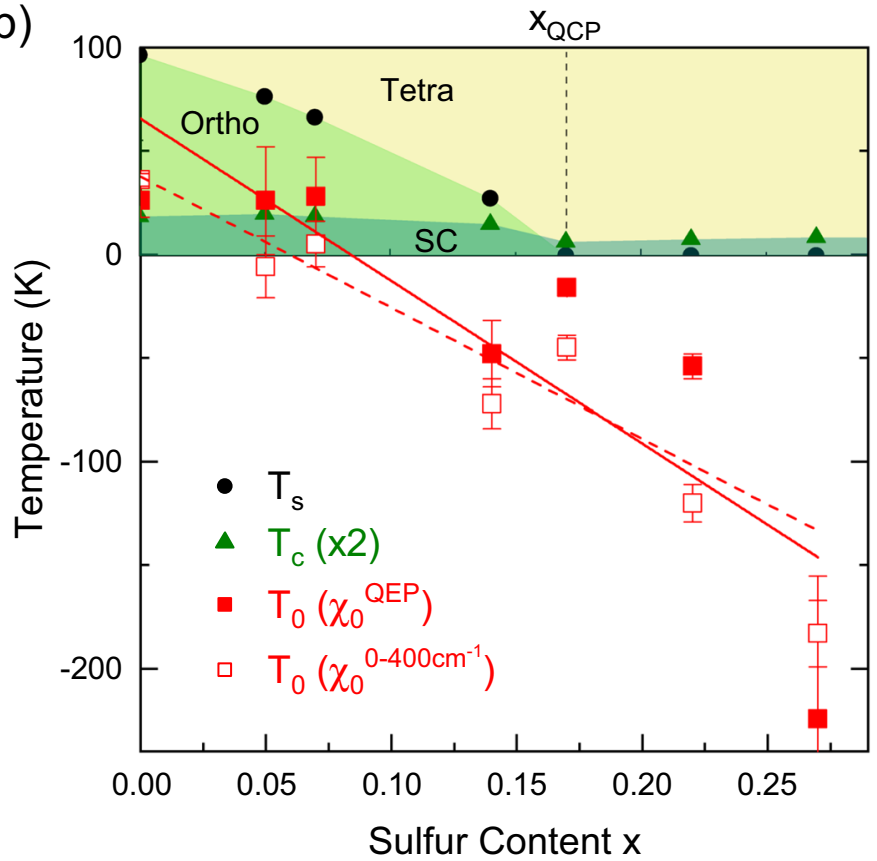

Fig. 4 Curie-Weiss analysis of the critical nematic susceptibility. a Curie-Weiss analysis of the critical inverse nematic susceptibility component $X_{0}^{\mathrm{QEP}}$. Note that only data above $T_{s}$ are shown for low $x$ compositions. b Phase diagram $x-T$ of FeSe $e_{1-x} S_{x}$ showing the evolution of the Curie-Weiss nematic transition temperature $T_{0}$ extracted from the analysis in (a) (full square). The error bars for $T_{0}$ correspond to standard errors of the Curie-Weiss fits. Also shown in open squares are the $T_{0}$ values extracted from a Curie-Weiss analysis of the $X_{0}$ extracted without any fitting by performing a Kramers-Kronig integration of the raw data restricted to energies below $400 \mathrm{~cm}^{-1}$ (see Supplementary Fig. 6). The lines are linear fits of $T_{0}(x)$ values.

temperatures we do not believe it is associated to the modulated orbital order conjectured in ${ }^{56}$. A suppression of the nematic fluctuations, and thus the QEP, is qualitatively expected in the nematic ordered phase. Still the strong suppression of the QEP observed could also be reinforced by an orbital transmutation effect below $T_{s}$, whereby the hole pocket becomes essentially single component in orbital space inducing strong vertex corrections that further suppress the $B_{1 \mathrm{~g}}$ Raman response in the nematic phase ${ }^{57}$.

Focusing now on the critical behavior of the QEP component, we display in Fig. 4a $\frac{1}{x_{0}^{\text {OEP }}}$ as a function of $x$ and $T$. We have added two additional compositions where spectra were taken in a more limited spectral range, allowing only the extraction of the QEP component (see Supplementary Fig. 4). Assuming a mean-field Curie-Weiss temperature dependence, we can extract the corresponding nematic Curie-Weiss temperature $T_{0}$ whose $x$ dependence is shown in Fig. 4b. We note that a Curie-Weiss temperature dependence does not necessarily imply that local nematic degrees of freedom are assumed. Indeed, it has been shown both in the context of itinerant magnetism and electronic nematicity that susceptibilities following Curie-Weiss law can be obtained even in purely itinerant but interacting electron models $s^{30,63}$. The evolution of $T_{0}$ extracted directly from the raw data using Kramer-Kronig relations restricted to energies below

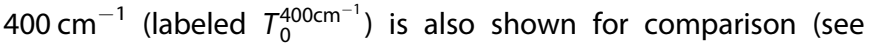
Supplementary Fig. 6). While less transparent, this determination has the advantage of not relying on any assumption about the functional form of the low energy part of the spectrum. Consistently with low energy QEP part of the $B_{1 \mathrm{~g}}$ spectrum being more critical, $T_{0}^{400 \mathrm{~cm}^{-1}}$ tend to be lower than $T_{0}^{\text {QEP }}$ determined from the QEP part only. Note also that even lower $T_{0}$ are obtained if the full temperature dependent part of the spectra, thus including both QEP and HE contributions to $X_{0}$, is integrated from 0 to $2000 \mathrm{~cm}^{-1}$ (see Supplementary Figs. 6 and 8). Since our focus is on the impact of nematic criticality on low energy physics which governs both SC and transport, we believe that it is the behavior of the low energy critical QEP that is the most relevant for the present discussion. Therefore we restrict our discussion to $T_{0}^{\mathrm{QEP}}$ and $T_{0}^{400 \mathrm{~cm}^{-1}}$ below.

For both determinations, $T_{0}$ decreases with $x$ tracking $T_{s}$ but stays significantly below it (Fig. 4b). The difference between $T_{s}$ and $T_{0}$ is a consequence of the finite electron-lattice coupling as first discussed by Kontani and Yamakawa ${ }^{64}$. Because the nematic susceptibility $X_{0}$ extracted from Raman spectra is in the dynamical limit ( $k=0$ and then $\omega=0$, while the opposite is required for a thermodynamical susceptibility), the symmetry allowed coupling between electronic nematic fluctuations and the soft orthorhombic acoustical phonon vanishes, leaving only the contribution from the bare electronic-only nematic susceptibility ${ }^{50}$. In this picture, $T_{0}$ represents the mean-field nematic transition temperature in the absence of the lattice. By contrast in the opposite thermodynamical or static limit, the electron-lattice comes into play and stabilizes the nematic transition at $T_{s}>T_{0}$. We note that such a picture was validated by comparing the temperature dependence of the Raman nematic susceptibility and the shear modulus in both $\mathrm{Ba}\left(\mathrm{Fe}_{1-x} \mathrm{CO}_{x}\right)_{2} \mathrm{As}_{2}$ and $\mathrm{FeSe}_{1-x} \mathrm{~S}_{x}{ }^{50,52}$. In $\mathrm{FeSe}_{1-x} \mathrm{~S}_{x}$, the difference between $T_{s}$ and $T_{0}$ is significant for the four compositions showing nematic order, ranging from 45 to $75 \mathrm{~K}$, depending on the method used to extract $T_{0}$. An unavoidable consequence of this large difference is a significant difference in the location of the associated critical points: the bare electronic nematic QCP extrapolates at $x_{\mathrm{QCP}}^{0} \sim 0.07( \pm 0.04)$, but the thermodynamical QCP is located at $x_{\mathrm{QCP}} \sim 0.17( \pm 0.02)$. We stress that the bare nematic QCP is only fictitious. Still, its location is paramount since as we discuss below, it ultimately governs the impact of the critical nematic fluctuations on low energy properties such as SC pairing and transport lifetime. 


\section{DISCUSSION}

As stated above, the $x$ evolution of $T_{0}$ points to a significant impact of lattice effects on nematic QCP physics. In order to quantify it, we introduce the ratio $r_{0}=\frac{k_{B}\left(T_{S}-T_{0}\right)}{E_{F}}$ where $E_{F}$ is the Fermi energy ${ }^{44} \cdot r_{0}=0$ signifies the absence of shift of the QCP. On the other hand, $r_{0} \sim 1$ indicates a strong impact of the lattice on the QCP that can manifest itself by, e.g., a restoration of Fermi liquid behavior above the QCP ${ }^{44,46}$. For pure FeSe, the hole and electron pocket are small, resulting in small $E_{F}$ of about $20 \mathrm{meV}$ for each $x z / y z$-derived electron pockets according to ARPES ${ }^{42}$. Adding $S$ only results in the appearance of a small inner-hole pocket above $E_{F}$, but does not change appreciably the size of the other pockets, at least below $x \sim 0.17$. Taking $20 \mathrm{meV}$ as an upper bound for $E_{F}$ for all $\mathrm{FeSe}_{1-x} \mathrm{~S}_{x}$, we plot $r_{0}$ versus $T_{s}$ as shown in Fig. 5 . Despite some scatter in the values of $r_{0}$, the plot suggests a significant value of $r_{0}$ extrapolated at the thermodynamic QCP where $T_{s}=0$ : from $r_{0}=0.35 \pm 0.15$. Also shown in Fig. 5 are values for $\mathrm{Ba} 122$ which are reduced compared to $\mathrm{FeSe}_{1-x} \mathrm{~S}_{x}$, but still significant ( 0.2).

Next, we discuss the consequences of our findings for the SC near the nematic QCP of FeSe ${ }_{1-x} \mathrm{~S}_{x}$. Theoretical works that ignored nemato-elastic coupling have argued that near a nematic QCP, the critical nematic fluctuations can lead to a boost in the SC $T_{c}$. On the other hand, once this coupling is taken into account it leads to a crossover Fermi liquid temperature scale $T_{\mathrm{FL}}=r_{0}^{3 / 2} E_{F}$ below which the critical nematic fluctuations are suppressed ${ }^{44}$. In the case of S-FeSe, we estimate $T_{\mathrm{FL}} \sim 55( \pm 25) \mathrm{K}$. Since $T_{\mathrm{FL}}>T_{\mathrm{c}} \sim 10 \mathrm{~K}$ in this system, it is unlikely that the critical nematic fluctuations play a role in the pairing in $\mathrm{FeSe}_{1-x} \mathrm{~S}_{x}$. For such a situation Labat et al. ${ }^{24}$ found that significant $T_{c}$ enhancement at the QCP occurs only if the nemato-elastic coupling is weak enough such that $r_{0}<$ $(U / V)^{2}$, where $U$ is an effective interaction in the nematic channel and $V$ is an effective SC pairing attraction due to, e.g., spin fluctuations. For Fe SC, we expect $V>U$ since pairing is dominated by spin fluctuations away from QCP. While there is currently no realistic theoretical evaluation of $V$ and $U$ for FeSe, since in practice no $T_{c}$ boost has been noted in $\mathrm{S}-\mathrm{FeSe}$, we conclude that the nemato-elastic coupling is significantly large with $r_{0}>\left(U / V^{2}\right.$.

The same analysis applied to $\mathrm{Ba}\left(\mathrm{Fe}_{1-x} \mathrm{Co}_{x}\right)_{2} \mathrm{As}_{2}$ gives $T_{\mathrm{FL}} \sim 40$ $( \pm 10) \mathrm{K}$, much closer to the actual $T_{c}$ observed in these systems. The above estimate suggests that Ba122 systems may be more

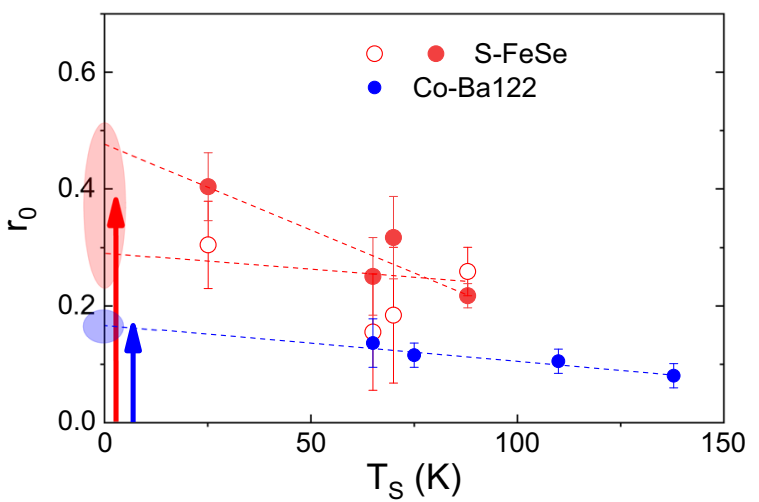

Fig. 5 Lattice-shifted nematic quantum criticality. Dimensionless nemato-electron-lattice coupling $r_{0}=\frac{k_{B}\left(T_{S}-T_{0}\right)}{E_{F}}$ as a function of $T_{s}$ for $\mathrm{FeSe}_{1-x} \mathrm{~S}_{X}$ and $\mathrm{Ba}\left(\mathrm{Fe}_{1-x} \mathrm{Co}_{x}\right)_{2} \mathrm{As}_{2}{ }^{50}$. The Fermi energy $E_{F}$ was taken to be 20 and $40 \mathrm{meV}$ for $\mathrm{FeSe}_{1-x} \mathrm{~S}_{x}$ and $\mathrm{Ba}\left(\mathrm{Fe}_{1-x} \mathrm{Co}_{x}\right)_{2} \mathrm{As}_{2}$, respectively. For FeSe, full (empty) circles correspond to $r_{0}$ values deduced from $T_{0}^{\text {QEP }}\left(T_{0}^{400 \mathrm{~cm}^{-1}}\right)$. By definition the origin of the graph corresponds to the location of the QCP in the absence of any coupling to the lattice $\left(T_{s}=T_{0}\right)$, the arrows illustrate the effect of the lattice on the QCP. The line is linear fit of $r_{0}\left(T_{s}\right)$ used to deduced $r_{0}\left(T_{s}=0\right)$, and the filled ovals illustrate their associated uncertainties. promising candidates for a significant effect of the nematic QCP on $T_{c}$. While the presence of a nearby magnetic QCP in most Ba122 complicates the matter, we note the recent observation of a significant enhancement of $T_{c}$ near a nematic QCP in $\mathrm{Ba}_{1-x} \mathrm{Sr}_{x} \mathrm{Ni}_{2} \mathrm{As}_{2}$ in the absence of any magnetic order ${ }^{19}$. Interestingly, band structure calculations predict a much larger Fermi surface and therefore a larger $E_{F}$ with respect to $\mathrm{BaFe}_{2} \mathrm{As}_{2}{ }^{65}$. This may possibly lead to a significantly reduced $r_{0}$, and thus much weaker lattice effects on the QCP of $\mathrm{Ba}_{1-x} \mathrm{Sr}_{x} \mathrm{Ni}_{2} \mathrm{Fe}_{2}$. Further investigations, in particular nematic susceptibility measurements, are needed to assess the electron-lattice coupling in this system and confirm this scenario.

Finally, we address the origin the noncritical HE peak component observed in the $B_{1 \mathrm{~g}}$ spectrum. As discussed above, it only appears in the $B_{1 \mathrm{~g}}$ nematic channel and its energy, around $400 \mathrm{~cm}^{-1}(50 \mathrm{meV})$, is only mildly temperature and $x$ dependent. A first possible interpretation is that it comes from interband transitions between the two hole bands centered at the $\Gamma$ point which are split by spin-orbit coupling ${ }^{57}$. In that case, however, we would expect the energy of the HE peak to be very close to the spin-orbit induced energy splitting ${ }^{57}$, which is estimated from ARPES measurements to be $20 \mathrm{meV}^{36}$, significantly below $50 \mathrm{meV}$. In addition, simple calculations of the interband Raman spectrum indicate that it should appear in both $B_{1 \mathrm{~g}}$ and $B_{2 \mathrm{~g}}$ symmetries with comparable intensity, again in disagreement with experiments (see Supplementary Note for Raman calculations of the contribution of interband transitions in the presence of spin-orbit coupling). This leads us to conclude that the HE peak is unlikely of interband origin.

As first argued by Baum et al. ${ }^{62}$ (see also ${ }^{66}$ ), the HE peak more likely arises from spin fluctuations associated to local moments. Local moments have been argued to be relevant for Fe SC due to Hund's coupling physics ${ }^{67-70}$, where electrons have both itinerant and localized, or "Janus face," character. Numerical calculations of the Raman response within a spin-1 Heisenberg localized spin model with bi-quadratic interactions with exchange parameters believed to be adequate for FeSe have indeed shown the presence of a paramagnon peak in the $B_{1 \mathrm{~g}}$ symmetry channel. Compared to two magnon excitations of ordered antiferromagnets, in FeSe the paramagnon is pushed below typical exchange energies due to magnetic frustration and the presence of both stripe-like and Néél-like AF fluctuations ${ }^{71}$. The presence of low-lying paramagnon excitations in FeSe has been confirmed by resonant inelastic X-ray scattering (RIXS) measurements, where a dispersive collective mode was observed, and interpreted as a paramagnon excitation ${ }^{72}$. The energy of the RIXS paramagnon peak near the zone center is close to $50 \mathrm{meV}$, similar to the Raman HE peak, further enforcing the interpretation of the HE peak in terms of local spin physics. We note that within the Fleury-Loudon formalism of Raman scattering in insulating magnets with nearest neighbors exchange, the $B_{1 \mathrm{~g}}$ Raman scattering operator for spin 1/ 2 is equivalent to the spin-nematic order parameter written in real space $O_{B_{1 g}}=P_{x}-P_{y}$ where $P_{a}=\sum_{r} \mathbf{S}_{r} \cdot \mathbf{S}_{r+a}{ }^{73}$. For spin 1 systems with bi-quadratic couplings, higher-order terms such as spin quadrupole will also come into play, and may contribute to the $B_{1 \mathrm{~g}}$ $\mathrm{HE}$ peak too ${ }^{74,75}$. If interpreted along these lines, the nematic susceptibility extracted from the HE component can be interpreted as the local spin-nematic component of the nematic susceptibility.

In conclusion, the picture emerging from these considerations is that of two distinct component in the nematic fluctuations spectrum in FeSe: one associated with itinerant carrier and displaying clear critical behavior as embodied by $\chi_{\mathrm{OEP}}^{0}$, and the other associated more correlated electrons and local moment physics which, while sizable, is less critical as observed for $\chi_{\mathrm{HE}}^{0}$. This suggests that the nematic instability is driven mainly by itinerant carriers. However, as we have shown the coupling of these fluctuations to the lattice most likely prevent them from playing a 
role in enhancing $T_{c}$ near the nematic QCP of FeSe ${ }_{1-x} \mathrm{~S}_{x}$. Other Fe SC systems with weaker nemato-elastic coupling, but ideally no other ordering, may provide a better platform to demonstrate this effect. Another open question is whether the local spin component has a role in the SC instability in FeSe. Our work suggests that this "Janus face" aspect of nematicity might be a generic feature of Hund's metals like FeSe.

\section{METHODS}

\section{Raman experiments}

Raman experiments have been carried out using a triple grating JY-T64000 spectrometer. Spectra below $500 \mathrm{~cm}^{-1}$ were obtained using subtractive mode and 1800 grooves $/ \mathrm{mm}$ gratings. High-energy spectra between 50 and $2500 \mathrm{~cm}^{-1}$ were obtained using a single stage spectrometer with a 600 grooves $/ \mathrm{mm}$ grating and long-pass edge filter to block the stray light. The spectrometer were equipped with a nitrogen cooled back illuminated CCD detector. All measurements were performed using the $532 \mathrm{~nm}$ excitation line from a diode pump solid-state laser. Measurement in $B_{1 \mathrm{~g}}$ symmetry channels was obtained using cross-incoming and -outgoing linear polarization oriented at 45 degrees of the Fe-Fe bonds, while $A_{1 \mathrm{~g}}+$ $B_{2 g}$ channel was obtained using parallel polarization at 45 degrees of the $\mathrm{Fe}-\mathrm{Fe}$ bonds. For several $x$ complimentary, measurements were performed in the $B_{2 g}$ symmetry channel using cross polarization along the $\mathrm{Fe}-\mathrm{Fe}$ bonds. The laser heating was estimated in situ by tracking the onset of elastic light scattering by orthorhombic domains across $T_{s}$ at different laser powers for $x=0, x=0.05$, and $x=0.14$ single crystals, yielding $1 \mathrm{~K} / \mathrm{mW}$ $( \pm 0.2)$ for all samples ${ }^{52}$. This value was taken for all the other samples. All the raw spectra have been corrected for the Bose factor and the instrumental spectral response. They are thus proportional to the imaginary part of the Raman response function $\chi^{\prime \prime}(\omega, T)$ in the corresponding symmetry channel. In order to compute the nematic susceptibility using Kramers-Kronig relation, the Raman susceptibility was interpolated from the lowest wavenumber measured $\left(10 \mathrm{~cm}^{-1}\right)$ to 0 using the QEP lineshape described in the main text.

\section{Samples}

The $x=0$ crystal was grown in Grenoble using the chemical vapor transport method based on the use of an eutectic mixture of $\mathrm{AlCl}_{3} / \mathrm{KCl}$ as described in ${ }^{76}$. The $x=0.05, x=0.07, x=0.14, x=0.17$, and $x=0.22$ single crystals were grown in Tohoku University by the molten salt flux methods ${ }^{77}$. Polycrystalline $\mathrm{FeSe}_{1-x} \mathrm{~S}_{x}$ for the single-crystal growth precursors were synthesized by the solid-state reaction methods ${ }^{78}$. Polycrystalline $\mathrm{FeSe}_{1-x} \mathrm{~S}_{x}$ and $\mathrm{KCl} / \mathrm{AlCl}_{3}$ were mixed up inside the Ar grove box with the molar ratio of 1:12 and were sealed in the quartz tube under the vacuum condition of $10^{-2} \mathrm{~Pa}$. The quartz ampoule was heated using the tube furnace. The temperature of hot and cold positions was kept at $390^{\circ} \mathrm{C}$ and $240^{\circ} \mathrm{C}$, respectively. After $\sim 10$ days, single crystals were grown around the cold part of the quartz tube. The $x=0.27$ crystal was grown in Ames out of an eutectic mix of $\mathrm{KCl} / \mathrm{AlCl}_{3}$ salts as described $\mathrm{in}^{79}$. Details about the growth and characterization of $x=0.27$ single crystals from the same batch can also be found in ${ }^{43}$. Single crystals from the same batch were characterized by transport measurements yielding first estimates of $T_{s}$ and $T_{c}$. The measured crystals were further characterized by SQUID magnetometry to obtain their $T_{c}$, by EDS and XRD to obtain their sulfur content $x$.

XRD data on $\mathrm{FeSe}_{1-x} \mathrm{~S}_{x}$ single crystals were collected at $295 \mathrm{~K}$ on a STOE imaging plate diffraction system (IPDS-2T) using Mo $K_{a}$ radiation. All accessible symmetry-equivalent reflections $(\approx 4500)$ were measured up to a maximum angle of $2 \Theta=65 \mathrm{deg}$. The data were corrected for Lorentz, polarization, extinction, and absorption effects. Using SHELXL ${ }^{80}$ and JANA2006 ${ }^{81}$, around 101 averaged symmetry-independent reflections $(I>2 \sigma)$ have been included for the refinements in space group $P 4 / \mathrm{nmm}$. The refinements converged quite well and the somewhat increased reliability factors (see GOF, $R_{1}$, and $w R_{2}$ in the Table) and uncertainties for the atomic positions and the ADPs result from the significant mosaic spread often observed for $\mathrm{FeSe}_{1-x} \mathrm{~S}_{x}$ samples. Results for $x=0.12,0.22$, and 0.27 are shown in Supplementary Table 1 as representatives.

In general, both EDS and X-ray yielded consistent results within $20 \%$ for $x$ except for the highest $S$ content $x=0.27$. We note that EDS is only a semi-quantitative measure of $x$ unless the element-specific $X$-ray yield is quantitatively calibrated for the given instrument. Therefore XRD data were trusted in most case, except for $x=0.14$ where the value of $T_{s}$ under the laser spot $(25 \mathrm{~K})$ indicates a slightly higher $\mathrm{S}$ content than the XRD value $(x=0.12(3))$.

In addition, $T_{s}$ was estimated in situ on the same single crystals by monitoring the onset for elastic light scattering by orthorhombic domains at very small powers $(0.1 \mathrm{~mW})$ for $x=0, x=0.05$, and $x=0.14$. Except for $x=0.07, T_{s}$ value quoted in the text were extracted from this method. For $x=0.07$, transport value from samples from the same batch was taken (see Supplementary Fig. 1).

\section{DATA AVAILABILITY}

All data generated or analyzed during this study are included in this published article (and its Supplementary Information files).

Received: 9 November 2020; Accepted: 12 March 2021; Published online: 12 April 2021

\section{REFERENCES}

1. Lohneysen, H. V., Rosch, A., Vojta, M. \& Wolfle, P. Fermi-liquid instabilities at magnetic quantum phase transitions. Rev. Mod. Phys. 79, 1015-1075 (2007).

2. Shibauchi, T., Carrington, A. \& Matsuda, Y. Quantum critical point lying beneath the superconducting dome in iron-pnictides. Annu. Rev. Condens. Matter Phys. 5, 113-135 (2014).

3. Taillefer, L. Scattering and pairing in high-T $\mathrm{T}_{c}$ cuprates. Annu. Rev. Condens. Matter Phys. 1, 51-70 (2010).

4. Monthoux, P., Pines, D. \& Lonzarich, G. G. Superconductivity without phonons. Nature 450, 1177-1183 (2007).

5. Metlitski, M. A., Mross, D. F., Sachdev, S. \& Senthil, T. Cooper pairing in non-Fermi liquids. Phys. Rev. B 91, 115111 (2015).

6. Berk, N. R. \& Schrieffer, J. R. Effect of ferromagnetic spin correlations on superconductivity. Phys. Rev. Lett. 17, 433-435 (1966).

7. Moriya, T. \& Ueda, K. Spin fluctuations and high-temperature superconductivity. Adv. Phys. 49, 555-606 (2000).

8. Fradkin, E., Kivelson, S. A., Lawler, M. J., Eisenstein, J. P. \& Mackenzie, A. P. Nematic Fermi fluids in condensed matter physics. Annu. Rev. Condens. Matter Phys. 1, 153-178 (2010).

9. Fernandes, R. M., Chubukov, A. V. \& Schmalian, J. What drives nematic order in iron-based superconductors? Nat. Phys. 10, 97-104 (2014).

10. Kuroki, K. et al. Unconventional pairing originating from the disconnected Fermi surfaces of superconducting LaFeAsO ${ }_{1-x} \mathrm{~F}_{x}$. Phys. Rev. Lett. 101, 087004 (2008).

11. Mazin, I. I. et al. Unconventional superconductivity with a sign reversal in the order parameter of $\mathrm{LaFeAsO}_{1-x} \mathrm{~F}_{x}$. Phys. Rev. Lett. 101, 057003 (2008).

12. Chu, J.-H., Kuo, H.-H., Analytis, J. G. \& Fisher, I. R. Divergent nematic susceptibility in an iron arsenide superconductor. Science 337, 710-712 (2012).

13. Fernandes, R. M. \& Millis, A. J. Nematicity as a probe of superconducting pairing in iron-based superconductors. Phys. Rev. Lett. 111, 127001 (2013).

14. Gallais, Y., Chauviere, L., Paul, I. \& Schmalian, J. Nematic resonance in the Raman response of iron-based superconductors. Phys. Rev. Lett. 116, 017001 (2016).

15. Hosoi, S. et al. Nematic quantum critical point without magnetism in $\mathrm{FeSe}_{1-x} \mathrm{~S}_{x}$ superconductors. Proc. Natl Acad. Sc. 113, 8139-8143 (2016).

16. Kuo, H. H., Chu, J.-H., Palmstrom, J. C., Kivelson, S. A. \& Fisher, I. R. Ubiquitous signatures of nematic quantum criticality in optimally doped Fe-based superconductors. Science 352, 958-962 (2016).

17. Wang, $C$. G. et al. Electron mass enhancement near a nematic quantum critical point in $\mathrm{NaFe}_{1-x} \mathrm{Co}_{x}$ As. Phys. Rev. Lett. 121, 167004 (2018).

18. Klein, A., Wu, Y.-M. \& Chubukov, A. V. Multiple intertwined pairing states and temperature-sensitive gap anisotropy for superconductivity at a nematic quantum-critical point. npj Quant. Mater. 4, 55 (2019).

19. Eckberg, C. et al. Sixfold enhancement of superconductivity in a tunable electronic nematic system. Nat. Phys. 16, 346-350 (2020).

20. Hong, X. et al. Evolution of the nematic susceptibility in $\mathrm{LaFe}_{1-x} \mathrm{Co}_{x}$ AsO. Phys. Rev. Lett. 125, 067001 (2020).

21. Yamase, H. \& Zeyher, R. Superconductivity from orbital nematic fluctuations. Phys. Rev. B 88, 180502 (2013).

22. Maier, T. A. \& Scalapino, D. J. Pairing interaction near a nematic quantum critical point of a three-band $\mathrm{CuO}_{2}$ model. Phys. Rev. B 90, 174510 (2014).

23. Lederer, S., Schattner, Y., Berg, E. \& Kivelson, S. A. Enhancement of superconductivity near a nematic quantum critical point. Phys. Rev. Lett. 114, 097001 (2015).

24. Labat, D. \& Paul, I. Pairing instability near a lattice-influenced nematic quantum critical point. Phys. Rev. B 96, 195146 (2017).

25. Boehmer, A. E. \& Kreisel, A. Andreas nematicity, magnetism and superconductivity in FeSe. J. Phys. Condens. Mat. 30, 023001 (2018). 
26. Coldea, A. I. \& Watson, M. D. The key ingredients of the electronic structure of FeSe. Ann. Rev. Condens. Matter Phys. 9, 125-146 (2018).

27. Glasbrenner, J. K. et al. Effect of magnetic frustration on nematicity and superconductivity in iron chalcogenides. Nat. Phys. 11, 953-958 (2015).

28. Wang, F., Kivelson, S. A. \& Lee, D.-H. Nematicity and quantum paramagnetism in FeSe. Nat. Phys. 11, 959-963 (2015).

29. Chubukov, A. V., Khodas, M. \& Fernandes, R. M. Magnetism, superconductivity, and spontaneous orbital order in iron-based superconductors: which comes first and why? Phys. Rev. X 6, 041045 (2016).

30. Yamakawa, Y., Onari, S. \& Kontani, H. Nematicity and magnetism in FeSe and other families of Fe-based superconductors. Phys. Rev. X 6, 021032 (2016).

31. Fanfarillo, L., Benfatto, L. \& Valenzuela, B. Orbital mismatch boosting nematic instability in iron-based superconductors. Phys. Rev. B 97, 121109 (2018).

32. Bendele, $M$. et al. Pressure induced static magnetic order in superconducting FeSe $_{1-x}$. Phys. Rev. Lett. 104, 087003 (2010).

33. Sun, J. P. et al. Dome-shaped magnetic order competing with high-temperature superconductivity at high pressures in FeSe. Nat. Comm. 7, 12146 (2016).

34. Kothapalli, K. et al. Strong cooperative coupling of pressure-induced magnetic order and nematicity in FeSe. Nat. Comm. 7, 12728 (2016).

35. Massat, P. et al. Collapse of critical nematic fluctuations in FeSe under pressure. Phys. Rev. Lett. 121, 077001 (2018).

36. Watson, M. D. et al. Suppression of orbital ordering by chemical pressure in $\mathrm{FeSe}_{1-x} \mathrm{~S}_{x}$. Phys. Rev. B 92, 121108 (2015).

37. Urata, T. et al. Non-fermi liquid behavior of electrical resistivity close to the nematic critical point in $\mathrm{Fe}_{1-x} \mathrm{Co}_{x} \mathrm{Se}$ and $\mathrm{FeSe}_{1-y} \mathrm{~S}_{y}$. Preprint at http://arxiv.org/ abs/1608.01044 (2016).

38. Sprau, P. O. et al. Discovery of orbital-selective Cooper pairing in FeSe. Science 357, 75-80 (2017).

39. Hanaguri, T. et al. Two distinct superconducting pairing states divided by the nematic end point in $\mathrm{FeSe}_{1-x} \mathrm{~S}_{x}$. Sci. Adv. 4, 6419 (2018).

40. Sato, Y. et al. Abrupt change of the superconducting gap structure at the nematic critical point in $\mathrm{FeSe}_{1-x} \mathrm{~S}_{x}$. Proc. Natl Acad. Soc. 115, 1227-1231 (2018).

41. Benfatto, L., Valenzuela, B. \& Fanfarillo, L. Nematic pairing from orbital-selective spin fluctuations in FeSe. npj Quant. Mater. 3, 56 (2018).

42. Reiss, P. et al. Suppression of electronic correlations by chemical pressure from FeSe to FeS. Phys. Rev. B 96, 121103 (2017).

43. Wiecki, P. et al. Persistent correlation between superconductivity and antiferromagnetic fluctuations near a nematic quantum critical point in $\mathrm{FeSe}_{1-x} \mathrm{~S}_{x}$. Phys. Rev. B 98, 020507 (2018).

44. Paul, I. \& Garst, M. Lattice effects on nematic quantum criticality in metals. Phys. Rev. Lett. 118, 227601 (2017).

45. de Carvalho, V. S. \& Fernandes, R. M. Resistivity near a nematic quantum critical point: impact of acoustic phonons. Phys. Rev. B 100, 115103 (2019).

46. Reiss, P. et al. Quenched nematic criticality and two superconducting domes in an iron-based superconductor. Nat. Phys. 16, 89-94 (2020).

47. Licciardello, S. et al. Electrical resistivity across a nematic quantum critical point Nature 567, 213-217 (2019).

48. Yamase, H. \& Zeyher, R. Raman scattering near a d-wave Pomeranchuk instability. Phys. Rev. B 83, 115116 (2011).

49. Gallais, Y. et al. Observation of incipient charge nematicity in $\mathrm{Ba}\left(\mathrm{Fe}_{1-x} \mathrm{Co}_{x} \mathrm{As}\right)_{2}$ single crystals. Phys. Rev. Lett. 111, 267001 (2013).

50. Gallais, Y. \& Paul, I. Charge nematicity and electronic Raman scattering in ironbased superconductors. C. R. Phys. 17, 113 (2016).

51. Thorsmølle, V. K. et al. Critical quadrupole fluctuations and collective modes in iron pnictide superconductors. Phys. Rev. B 93, 054515 (2016).

52. Massat, P. et al. Charge-induced nematicity in FeSe. Proc. Natl Acad. Soc. 113, 9177-9181 (2016).

53. Klein, A., Lederer, S., Chowdhury, D., Berg, E. \& Chubukov, A. V. Dynamical susceptibility near a long-wavelength critical point with a nonconserved order parameter. Phys. Rev. B 97, 155115 (2018).

54. Auvray, N. et al. Nematic fluctuations in the cuprate superconductor $\mathrm{Bi}_{2} \mathrm{Sr}_{2} \mathrm{CaCu}_{2} \mathrm{O}_{8}$. Nat. Comm. 10, 5209 (2019).

55. Adachi, T., Nakajima, M., Gallais, Y., Miyasaka, S. \& Tajima, S. Superconducting gap and nematic resonance at the quantum critical point observed by Raman scattering in BaFe2 $\left(\mathrm{As}_{1-x} \mathrm{P}_{x}\right)_{2}$. Phys. Rev. B 101, 085102 (2020).

56. Zhang, W. L. et al. Stripe quadrupole order in the nematic phase of $\mathrm{FeSe}_{1-x} \mathrm{~S}_{x}$ Preprint at http://arxiv.org/abs/1710.09892 (2017).

57. Udina, M., Grilli, M., Benfatto, L. \& Chubukov, A. V. Raman response in the nematic phase of FeSe. Phys. Rev. Lett. 124, 197602 (2020).

58. Wang, X. \& Berg, E. Low frequency Raman response near Ising-nematic quantum critical point: a memory matrix approach. Preprint at http://arxiv.org/abs/ 2011.01818 (2020).

59. Khodas, M. \& Levchenko, A. Raman scattering as a probe of nematic correlations. Phys. Rev. B 91, 235119 (2015).
60. Karahasanovic, U. et al. Manifestation of nematic degrees of freedom in the Raman response function of iron pnictides. Phys. Rev. B 92, 075134 (2015).

61. Kretzschmar, F. et al. Critical spin fluctuations and the origin of nematic order in $\mathrm{Ba}\left(\mathrm{Fe}_{1-x} \mathrm{Co}_{x}\right) 2 \mathrm{As} 2$. Nat. Phys. 12, 560-563 (2016).

62. Baum, A. et al. Frustrated spin order and stripe fluctuations in FeSe. Commun Phys. 2, 14 (2019).

63. Moriya, T. Recent progress in the theory of itinerant electron magnetism. J. Magn. Magn. Mater. 14, 1-46 (1979).

64. Kontani, H. \& Yamakawa, Y. Linear response theory for shear modulus $C_{6} 6$ and Raman quadrupole susceptibility: evidence for nematic orbital fluctuations in $\mathrm{Fe}$ based superconductors. Phys. Rev. Lett. 113, 047001 (2014).

65. Subedi, A. \& Singh, D. J. Density functional study of $\mathrm{BaNi}_{2} \mathrm{As}_{2}$ : electronic structure, phonons, and electron-phonon superconductivity. Phys. Rev. B 78, 132511 (2008).

66. Glamazda, A., Lemmens, P., Ok, J. M., Kim, J. S. \& Choi, K.-Y. Dichotomic nature of spin and electronic fluctuations in FeSe. Phys. Rev. B 99, 075142 (2019).

67. Yin, Z. P., Haule, K. \& Kotliar, G. Kinetic frustration and the nature of the magnetic and paramagnetic states in iron pnictides and iron chalcogenides. Nat. Mat. 10, 932-935 (2011).

68. Medici, L., Mravlje, J. \& Georges, A. Janus-faced influence of Hund's rule coupling in strongly correlated materials. Phys. Rev. Lett. 107, 256401 (2011).

69. Lanatà, N. et al. Orbital selectivity in Hund's metals: the iron chalcogenides. Phys. Rev. B 87, 045122 (2013).

70. Skornyakov, S. L., Anisimov, V. I., Vollhardt, D. \& Leonov, I. Effect of electron correlations on the electronic structure and phase stability of FeSe upon lattice expansion. Phys. Rev. B 96, 035137 (2017).

71. Ruiz, H. et al. Frustrated magnetism from local moments in FeSe. Phys. Rev. B 99, 125130 (2019).

72. Rahn, M. C. et al. Paramagnon dispersion in $\beta$-FeSe observed by Fe L-edge resonant inelastic X-ray scattering. Phys. Rev. B 99, 014505 (2019).

73. Fleury, P. A. \& Loudon, R. Scattering of light by one- and two-magnon excitations Phys. Rev. 166, 514-530 (1968).

74. Michaud, F., Vernay, F. \& Mila, F. Theory of inelastic light scattering in spin-1 systems: resonant regimes and detection of quadrupolar order. Phys. Rev. B 84 184424 (2011).

75. Wen-Jun, H. et al. Quantum transitions of nematic phases in a spin-1 bilinearbiquadratic model and their implications for FeSe. Phys. Rev. Res. 2, 023359 (2020).

76. Karlsson, S. et al. Study of high-quality superconducting FeSe single crystals: crossover in electronic transport from a metallic to an activated regime above 350 K Supercond. Sci. Technol. 28, 105009 (2015).

77. Urata, T. et al. Argument on superconductivity pairing mechanism from cobalt impurity doping in FeSe: spin $\left(\mathrm{s}^{+-}\right)$or orbital $\left(\mathrm{s}^{++}\right)$fluctuation. Phys. Rev. B 93 014507 (2016).

78. Mizuguchi, Y. et al. Substitution effects on FeSe superconductor. J. Phys. Soc. Jpn. 78, 074712 (2009).

79. Böhmer, A. E., Taufour, V., Straszheim, W. E., Wolf, T. \& Canfield, P. C. Variation of transition temperatures and residual resistivity ratio in vapor-grown FeSe. Phys. Rev. B 94, 024526 (2016).

80. Sheldrick, G. M. Crystal structure refinement with SHELXL. Acta Crystallogr. A 64, 112 (2008).

81. Petricek, V., Dusek, M. \& Palatinus, L. The crystallographic computing system JANA2006: general features. Z. Kristallogr. Cryst. Mater. 229, 345 (2014).

\section{ACKNOWLEDGEMENTS}

We thank A. V. Chubukov and T. Shibauchi for fruitful discussions. We also thank G. Wang for his assistance with the EDS measurements. We also thank Laboratoire de Physique des Solides, Université Paris Saclay for making their MPMS SQUID magnetometer available. Crystal growth and characterization in Ames was supported by the US Department of Energy (DOE), Office of Basic Energy Sciences, Division of Materials Sciences and Engineering. Ames Laboratory is operated for the US DOE by lowa State University under Contract No. DE-AC02-07CH11358. The contribution from M.M. was supported by the Karlsruhe Nano Micro Facility (KNMF).

\section{AUTHOR CONTRIBUTIONS}

S.C., P.M., and D.F. performed the Raman scattering experiments with the help of M.C., A.S., and Y.G. S.C. performed the data analysis and prepared the figures. T.U., Y.T., K.T., S.K., P.S., P.T., A.E.B., and P.C.C. grew the single crystals. S.C. performed EDS and SQUID magnetometry measurement and M.M. performed single crystals XRD to characterize the samples. I.P. provided theoretical insights. Y.G. supervised the project and wrote the paper with inputs from all the authors. 


\section{COMPETING INTERESTS}

The authors declare no competing interests.

\section{ADDITIONAL INFORMATION}

Supplementary information The online version contains supplementary material available at https://doi.org/10.1038/s41535-021-00336-3.

Correspondence and requests for materials should be addressed to Y.G.

Reprints and permission information is available at http://www.nature.com/reprints

Publisher's note Springer Nature remains neutral with regard to jurisdictional claims in published maps and institutional affiliations.
Open Access This article is licensed under a Creative Commons Attribution 4.0 International License, which permits use, sharing, adaptation, distribution and reproduction in any medium or format, as long as you give appropriate credit to the original author(s) and the source, provide a link to the Creative Commons license, and indicate if changes were made. The images or other third party material in this article are included in the article's Creative Commons license, unless indicated otherwise in a credit line to the material. If material is not included in the article's Creative Commons license and your intended use is not permitted by statutory regulation or exceeds the permitted use, you will need to obtain permission directly from the copyright holder. To view a copy of this license, visit http://creativecommons. org/licenses/by/4.0/.

(c) The Author(s) 2021 